\title{
Fimasartan, an angiotensin II receptor antagonist, ameliorates an in vivo zebrafish model of heart failure
}

\author{
Hailian Quan ${ }^{1, *}$, Gyu Chul Oh ${ }^{2,3,}$, , Seung Hyeok Seok ${ }^{1}$, and Hae-Young Lee ${ }^{2,3}$
}

${ }^{1}$ Department of Microbiology and Immunology, Institute of Endemic Disease, ${ }^{2}$ Department of Internal Medicine, Seoul National University College of Medicine, Seoul; ${ }^{3}$ Department of Internal Medicine, Seoul National University Hospital, Seoul, Korea

Received: January 24, 2019

Revised : May 30, 2019

Accepted: June 4, 2019

\section{Correspondence to}

Hae-Young Lee, M.D.

Department of Internal

Medicine, Seoul National

University Hospital, 101 Daehak-

ro, Jongno-gu, Seoul 0308o,

Korea

Tel: +82-2-2072-0698

Fax: +82-2-3674-0805

E-mail: hylee612@snu.ac.kr

https://orcid.org/0000-0002-

9521-4102

Seung Hyeok Seok, Ph.D.

Department of Microbiology and Immunology, Seoul National University College of Medicine, 103 Daehak-ro, Jongno-gu, Seoul 03080, Korea

Tel: +82-2-740-8302

Fax: $+82-2-763-5206$

E-mail: lamseok@snu.ac.kr

https://orcid.org/0000-0002-

4315-0688

*These authors contributed equally to this work.

Background/Aims: Angiotensin II in the failing heart initially helps to maintain cardiac output and blood pressure, but ultimately accelerates its deterioration. In this study, we established a model of arrhythmia-induced heart failure (HF) in zebrafish and investigated the role of renin-angiotensin-aldosterone system (RAAS) modulation by using an angiotensin II type 1 receptor blocker, fimasartan, through the assessment of cellular and physiologic responses, morbidity, and mortality.

Methods: HF was induced in zebrafish larvae by exposure to $20 \mu \mathrm{M}$ terfenadine. Morphologic, physiologic, and functional parameters were assessed in the presence or absence of fimasartan treatment.

Results: Zebrafish exposed to terfenadine showed marked dilatation of the ventricle and reduced systolic function. Treatment with terfenadine was associated with 10 -fold higher expression of atrial natriuretic peptide $(p<0.001$ vs. vehicle), increased p53 mRNA expression, and chromatin fragmentation in the TUNEL assay, all of which were significantly reduced by fimasartan treatment. Moreover, fimasartan improved fractional shortening (terfenadine + fimasartan $16.9 \% \pm 3.1 \%$ vs. terfenadine + vehicle $11.4 \% \pm 5.6 \%, p<0.05$ ) and blood flow (terfenadine + fimasartan $479.1 \pm 124.1 \mathrm{~nL} / \mathrm{sec}$ vs. terfenadine + vehicle $273.0 \pm 109.0 \mathrm{~nL} / \mathrm{sec}, p<0.05)$. Finally, treatment with fimasartan remarkably reduced mortality (terfenadine + fimasartan $36.0 \%$ vs. terfenadine + vehicle $96.0 \%$, $p<0.001$ ).

Conclusions: Fimasartan effectively protected against the progression of HF in zebrafish by improving hemodynamic indices, which improved survival. A reduction in apoptotic cell death and an improvement in hemodynamics may be the mechanisms behind these effects. Further human studies are warranted to evaluate the possible role of fimasartan in the treatment of $\mathrm{HF}$.

Keywords: Heart failure; Angiotensin receptor blockers; Renin angiotensin system; Zebrafish; Terfenadine

\section{INTRODUCTION}

Heart failure (HF) affects approximately 26 million people worldwide, with more than one million patients hospitalized every year in the United States and Europe [1]. In the United States, HF accounted for one out of nine deaths in 
2013 [2]. Owing to the aging population, the prevalence of $\mathrm{HF}$ is increasing [3]. Despite the development of new pharmacotherapy agents for HF, it remains a serious health problem and is associated with high mortality and significant medical costs $[4,5]$.

The goals of therapy for patients with advanced HF are to improve survival, slow disease progression, and alleviate symptoms. Renin-angiotensin-aldosterone system (RAAS) blockers, such as angiotensin converting enzyme (ACE) inhibitors and angiotensin receptor blockers (ARBs), are commonly used to achieve these goals; they decrease preload and make it easier for the heart to pump blood. However, the use of ACE inhibitors is limited by the common side effects, such as cough; therefore, ARBs are potential alternative treatments [6].

Fimasartan is the ninth and the most recent ARB to be approved by the Korean Food and Drug Association as an antihypertensive agent [7-9]. In previous studies, fimasartan was shown to exert protective effects in animal models of myocardial ischemia: the suppression of apoptosis [10] and the prevention of intimal thickening and plaque rupture [11]. Furthermore, fimasartan has been reported to prevent doxorubicin-induced cardiomyopathy and improve survival [12]. Therefore, fimasar$\tan$ is regarded as a potential candidate for the attenuation of HF.

Recently, in vivo chemical screening in a zebrafish model of HF has emerged as a rapid and efficient method to identify lead compounds that modulate specific biological processes $[13,14]$. We have also previously reported a zebrafish model of dilated cardiomyopathy induced by brief treatment with terfenadine [15].

In this study, we hypothesized that treatment with fimasartan exerted protective effects against the development of $\mathrm{HF}$ and improved survival through the prevention of apoptotic cell death in a terfenadine-induced in vivo zebrafish model of HF.

\section{METHODS}

\section{Zebrafish husbandry and breeding}

Zebrafish (Danio rerio) embryos were maintained in egg water at $28.5^{\circ} \mathrm{C}$ in an automatic circulating tank system in accordance with instructions from The Zebrafish Book [16]. Adult fish were maintained at $28^{\circ} \mathrm{C}$ under a 14:10 hours light:dark cycle, and were fed three times daily with Artemia (INVE, Dendermonde, Belgium). To visualize the heart chambers, a transgenic strain of zebrafish larvae that expresses green fluorescent protein (GFP) exclusively in the cardiac myosin light chain 2 (cmlc2) $[\mathrm{Tg}$ (cmlc2:gfp)] was used [17]. $\mathrm{Tg}(\mathrm{cmlc2:gfp})$ lines were kindly provided by the zebrafish Organogenesis Mutant Bank in Korea. All animal study protocols were approved by the Institutional Animal Care and Use Committee of Seoul National University (SNU-150330-3).

\section{Chemical treatment of zebrafish}

To maintain the optical clarity of embryos, the egg water was supplemented with $0.003 \%$ 1-phenyl-2-thiourea (Sigma, St. Louis, MO, USA) at 24 hours post-fertilization (dpf). For all experiments excluding survival analysis, the larvae were transferred into 6-well microplates and egg water was replaced with a solution containing $20 \mu \mathrm{M}$ terfenadine (Sigma) or vehicle (0.1\% dimethyl sulfoxide [DMSO]) for 12 hours at 3 days dpf. After terfenadine treatment, fimasartan (Boryung Pharm Co. Ltd., Seoul, Korea) was added to the treatment group for 12 hours before the analysis was performed.

\section{Cardiac morphology}

After 12 hours treatment with fimasartan, wild-type zebrafish were anesthetized with $0.04 \%$ tricaine (Sig$\mathrm{ma}$ ) for 1 minute and images were obtained by using a stereomicroscope (M165 FC, Leica, Wetzlar, Germany) for morphologic observation. $\mathrm{Tg}$ (cmlcz:gfp) larvae were immersed in $0.15 \%$ low-melt agarose and transferred to a confocal dish to visualize the movement of cardiac chambers by using a fluorescence microscope (Leica $\mathrm{AF} 2000)$. The atrial area was calculated by using ImageJ graphical analysis software (National Institutes of Health, Bethesda, MD, USA). At minimum, five different larvae in each group were analyzed.

\section{Functional assessment}

Fractional shortening (FS) was calculated from the ventricular diastolic and systolic diameters, as measured from time-lapse images collected intervals of $0.16 \mathrm{sec}$ onds. Long-axis diameters were acquired from still images by using ImageJ software and FS was calculated from the following formula: FS $=(\mathrm{VDD}-\mathrm{VSD}) / \mathrm{VDD} \times$ $100 \%$ (VDD, ventricle diastolic diameter; VSD, ventricle 
systolic diameter).

The blood flow and time interval between heartbeats were also assessed. After exposure to the drug, the larvae were anesthetized with $0.04 \%$ tricaine for 1 minutes, transferred to a confocal dish, and visualization by using a stereomicroscope (Leica M165 FC) fitted with a highspeed video camera. The orientation of the larva was then adjusted very gently so that the larva lay on its side with its head to the left. First, the camera was positioned to capture the whole heart at a rate of 30 frames per seconds (fps), and then repositioned to capture the dorsal aorta and caudal to the swim bladder at 120 fps. The camera in both positions was independently focused on the respective regions of interest to ensure optimal image quality, and set to record simultaneously.

To determine atrioventricular (AV) synchrony, videos of the heart were analyzed by using MicroZebraLab version 3.5 (ViewPoint, Lyon, France). The arrhythmic rate was calculated by using the following formula: arrhythmic rate $(\%)=\mathrm{VBR} / \mathrm{ABR}$ (VBR, ventricular beat rate; $\mathrm{ABR}$, atrial beat rate). The blood flow videos were also analyzed by using ZebraBlood v1.3.2 (ViewPoint), which detects changes in heartbeat intervals.

\section{RNA isolation and quantitative real-time PCR}

Total mRNA was extracted from 20 larvae per treatment group by using TRIzol reagent (Invitrogen, Carlsbad, CA, USA) in accordance with the manufacturer's instructions. One microgram of isolated RNA was reverse-transcribed into cDNA by using M-MLV reverse transcriptase (Enzynomics, Daejeon, Korea). Quantitative PCR was performed by using SYBR Green qPCR Master Mix (Applied Biosystems, Foster City, CA, USA) and an ABI real-time PCR 7500 machine (Applied Biosystems). All samples were normalized to the mRNA expression of $\beta$-actin. The primers used were as follows: natriuretic peptide b (Nppb) forward primer: 5'-CAT GGG TGT TTT AAA GTT TCT CC-3'; Nppb reverse primer: 5'-CTT CAA TAT TTG CCG CCT TTA C-3'; p53 forward primer: 5'GGG CAA TCA GCG AGC AAA-3'; 253 reverse primer: 5'ACT GAC CTT CCT GAG TCT CCA-3'; $\beta$-actin forward primer: 5'-TGG TGA CCT GAC AGA CTA CCT GAT-3'; $\beta$-actin reverse primer: 5'-CGG ACA ATT TCT CTT TCG GCT GTG-3'.

\section{TUNEL assay}

The fragmentation of DNA in apoptotic cells was identified by the terminal deoxynucleotide transferase-mediated dUTP nick-end labeling (TUNEL) assay using the In Situ Cell Death Detection Kit, POD (Roche Diagnostics GmbH, Mannheim, Germany). Zebrafish larvae were fixed in $4 \%$ paraformaldehyde (PFA) overnight at $4{ }^{\circ} \mathrm{C}$. After fixation, excess $4 \%$ PFA was removed by five rinses in phosphate-buffered saline with Tween 20 (PBST), each of 5 minutes, and $100 \%$ methanol was added. The larvae were incubated at $-20^{\circ} \mathrm{C}$ for at least 30 minutes, and rehydrated in a graded methanol series $(75 \%, 50 \%, 25 \%$, and twice in $\% \%)$. The larvae were then incubated with proteinase $\mathrm{K}(50 \mu \mathrm{g} / \mathrm{mL}$ in $\mathrm{PBST})$ for 30 minutes at room temperature (RT), and excess proteinase $\mathrm{K}$ was removed by two rinses in PBST, each of 5 minutes. The larvae were re-fixed in $4 \%$ PFA for 20 minutes at RT and excess $4 \%$ PFA was removed by five rinses with PBST, each of 5 minutes, and then post-fixed by the application of a 2:1 mixture of pre-chilled ethanol and acetic acid for 10 minutes at $-20^{\circ} \mathrm{C}$. After three rinses with PBST, each of 5 minutes, the larvae were incubated for 2 hours at $37^{\circ} \mathrm{C}$ in labeling solution, which consisted of terminal deoxynucleotidyl transferase (TdT) and fluorescein-conjugated deoxynucleotide in buffer. After three rinses with PBST, each of 5 minutes, the larvae were incubated for $30 \mathrm{~min}$ utes in a converter-peroxidase (POD) in a humidified chamber at $37^{\circ} \mathrm{C}$. Finally, excess converter-POD was removed by three rinses with PBST, each of 5 minutes, and apoptotic cells were visualized by using metal-enhanced DAB (Dako, Carpinteria, CA, USA).

\section{Survival and motility assay}

For the survival assay, larvae at $3 \mathrm{dpf}$ were immersed in $20 \mu \mathrm{M}$ terfenadine for 12 hours, and $200 \mu \mathrm{M}$ fimasartan was added for 60 hours before analysis. For the motility assay, larvae at $3 \mathrm{dpf}$ were immersed in $20 \mu \mathrm{M}$ terfenadine for 24 hours and the medium was replaced with egg water containing $200 \mu \mathrm{M}$ fimasartan for 24 hours in the treatment group. The larvae were transferred to 6-well microplates in egg water and allowed to move freely at room temperature. The embryo positions were recorded every 0.5 second for 20 seconds to generate a trace.

\section{Statistical analysis}

Continuous variables were shown as the mean \pm stan- 

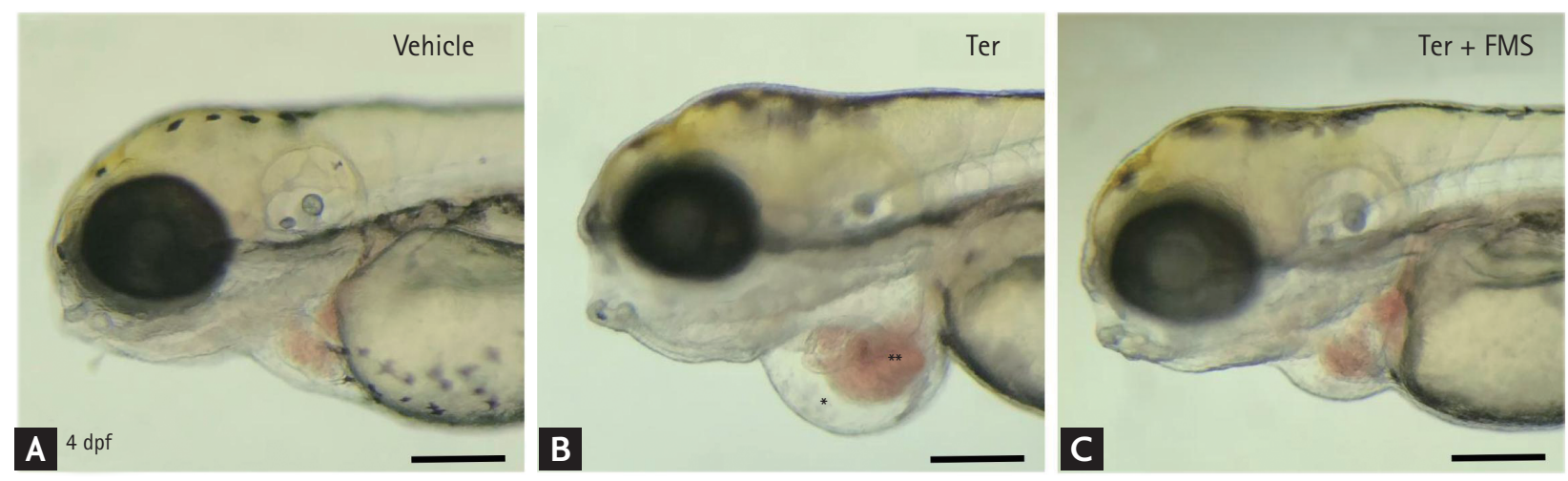

D
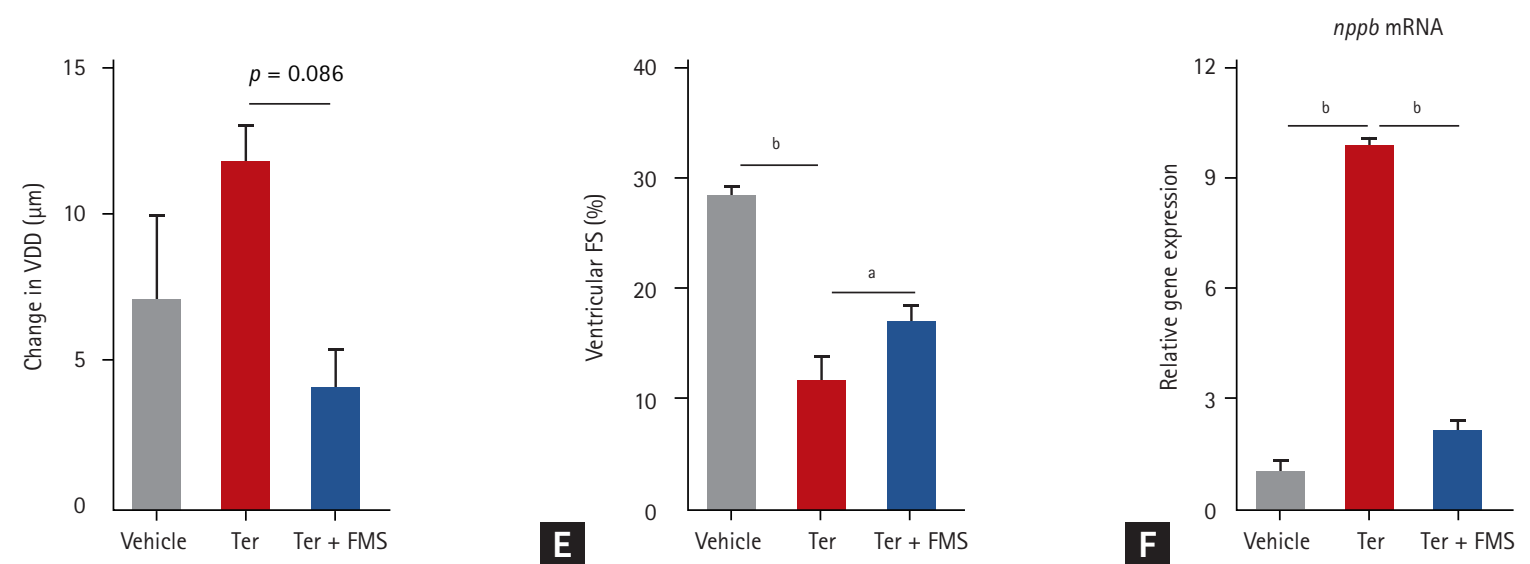

Figure 1. Fimasartan ameliorates heart failure in terfenadine-induced zebrafish larvae. (A-C) Microscopic images of zebrafish hearts. In comparison with (A) vehicle, (B) terfenadine-treated larvae show an increase in heart size, edema (*) and venous congestion $\left({ }^{* *}\right)$. (C) Treatment with fimasartan ameliorates changes brought on by terfenadine. (D) Changes in ventricular diastolic diameter and (E) ventricular FS ( $\mathrm{n}=5$ for each group). (F) Levels of $n p p b$ mRNA expression ( $\mathrm{n}=20$ for each group). Scale bar $=1 \mathrm{~mm}$. Error bars represent standard deviation. VDD, ventricular diastolic diameter; FS, fractional shortening; dpf, days post-fertilization; Ter, terfenadine; FMS, fimasartan; $n p p b$, natriuretic peptide b. ${ }^{a} p<0.05,{ }^{b} p<0.001$.

dard deviation and assessed by using the Student's $t$ test and analysis of variance (ANOVA). Categorical variables were presented as numbers and percentages and were assessed by using the chi-square test, with Kaplan-Meier analysis used for the comparison of survival. Statistical analyses were computed by using SPSS version 25 (IBM, Chicago, IL, USA) and figures were drawn by using an open-trial version of GraphPad Prism version 5 (GraphPad Software, San Diego, CA, USA). A p value of $<0.05$ was deemed statistically significant and is indicated in the figures by an asterisk; $p$ values of $<0.01$ and $<0.001$ are indicated by two and three asterisks, respectively.

\section{RESULTS}

\section{Fimasartan ameliorates terfenadine-induced HF in zebrafish}

Zebrafish larvae at $3 \mathrm{dpf}$ were treated with either 20 $\mu \mathrm{M}$ terfenadine or vehicle (0.1\% DMSO) for 12 hours. In the fimasartan treatment group, $200 \mu \mathrm{M}$ fimasartan was added and the larvae were incubated for 12 hours. Consistent with our previous study [15], zebrafish treated with terfenadine for 24 hours developed progressive $\mathrm{HF}$, with dilatation of the ventricle, venous congestion, and decreased ventricular contractility as measured by FS (Fig. 1A-1E). Nppb mRNA expression was higher in the terfenadine-treated larvae than in vehicle-treated larvae (Fig. $1 \mathrm{~F}$ ). These findings indicated that transient 

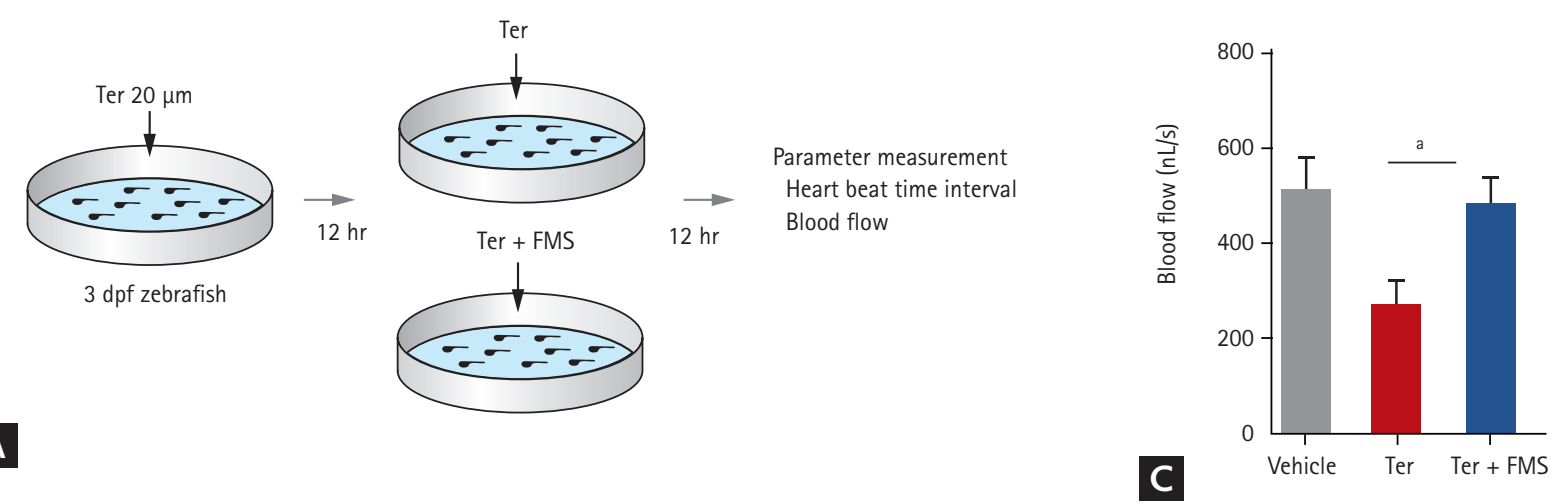

A
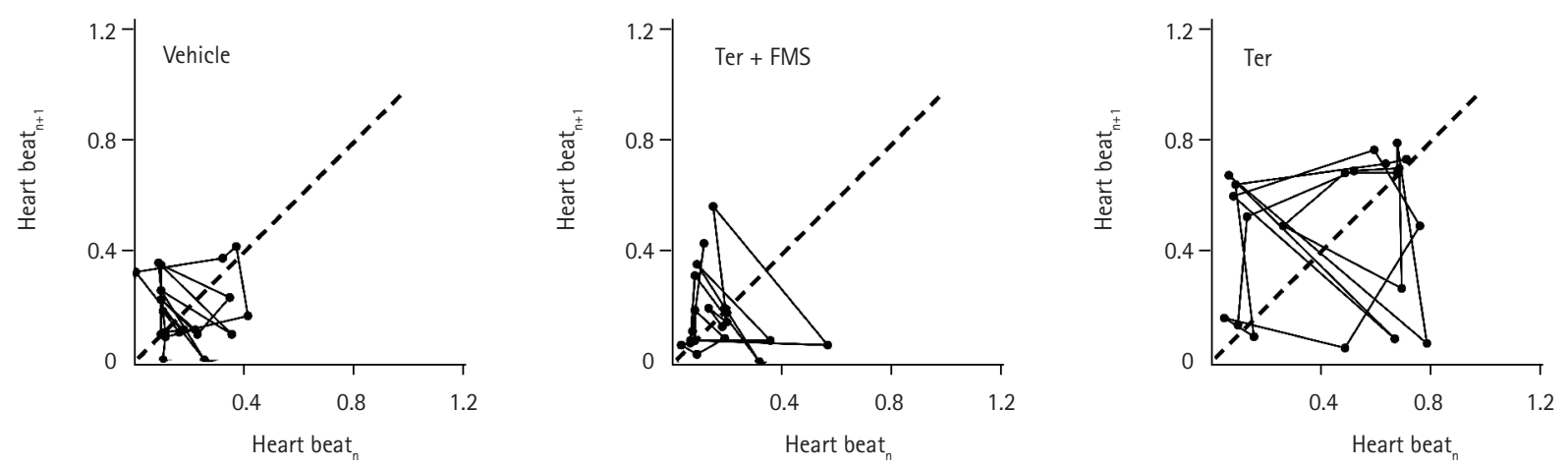

Figure 2. Fimasartan promotes recovery of cardiac output. (A) Study design. (B) Poincaré plot showing heart beat variations. (C) Blood flow velocity of zebrafish larvae ( $n=5$ for each group). Error bars represent standard deviation. Ter, terfenadine; dpf, days post-fertilization; FMS, fimasartan. ${ }^{\mathrm{a}} \mathrm{p}<0.05$.

treatment with terfenadine induced HF in zebrafish and reproduced the characteristics of human patients with $\mathrm{HF}$, such as dilatation of the cardiac chambers and decreased ventricular function.

Treatment with fimasartan reduced ventricular dilatation and $n p p b$ mRNA expression induced by terfenadine (Fig. $1 \mathrm{C}$ and $1 \mathrm{~F}$ ). Ventricular function, as assessed by FS, was also significantly preserved in the fimasartan treatment group compared with the terfenadine only group $(16.9 \% \pm 3.1 \%$ vs. $11.4 \% \pm 5.6 \%, p<0.05)$.

\section{Fimasartan promotes recovery of cardiac output}

To explore the physiologic effect of fimasartan in the zebrafish model of HF, hemodynamic parameters were measured, such as heartbeat interval and blood flow velocity (Fig. 2A). To assess the variability in heart rate, Poincaré plots were produced by using two consecutive time intervals between heartbeats. In control larvae treated with vehicle only, the data points in the plot were concentrated between 0.01 and 0.30 second, clus- tering at approximately 0.19 second. In contrast, terfenadine-treated larvae resulted in a dispersed plot, deviating from the control axis (in the range from 0.10 to 0.80 second). Heartbeat variability significantly increased from $0.19 \pm 0.12$ second in vehicle-treated larvae to 0.46 \pm 0.27 seconds in terfenadine-treated larvae $(p<0.001)$. Treatment with fimasartan markedly reduced the variability, to $0.16 \pm 0.13$ second $(p<0.001)$, with heartbeat intervals in the range from 0.08 to 0.40 second (Fig. $2 \mathrm{~B}$ ).

Blood flow velocity was lower in terfenadine-treated larvae than in vehicle-treated larvae, whereas fimasartan treatment preserved blood flow velocity. In detail, blood flow velocity in the terfenadine-treated larvae decreased from $507.0 \pm 170.6$ to $273.0 \pm 109.0 \mathrm{~nL} / \mathrm{sec}$, whereas treatment with $200 \mu \mathrm{M}$ fimasartan resulted in marked preservation of blood flow velocity, with a value of $479.1 \pm$ 124.1 nL/sec (Fig. 2C). The response of blood flow velocity to fimasartan treatment was also dose-dependent (Supplementary Fig. 1). These results indicated that fimasartan could reverse the variability in heartbeat and the 

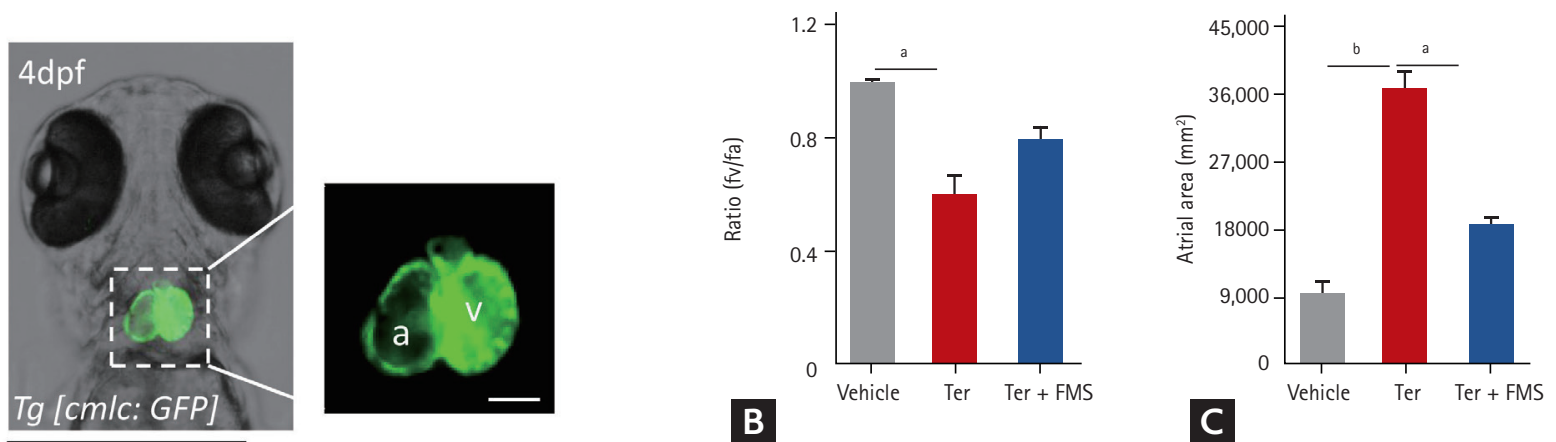

Vehicle

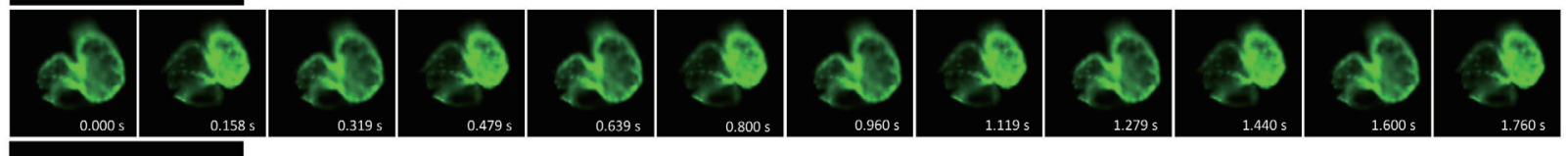

Ter

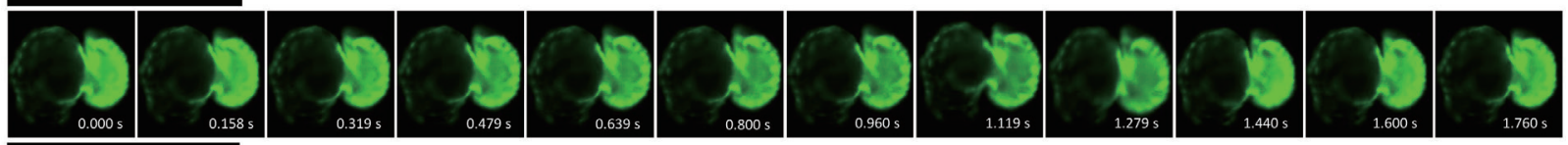

Ter + FMS

A

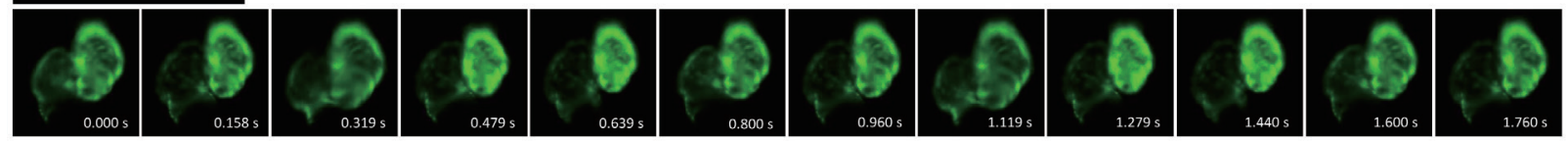

Figure 3. Fimasartan improves atrioventricular dyssynchrony. (A) Time-lapse images showing the dynamic heartbeat of zebrafish larvae. (B) Atrioventricular ratio of zebrafish larvae. (C) Atrial area of zebrafish larvae. Scale bar $=0.5 \mu \mathrm{m}$. Error bars represent standard deviation ( $\mathrm{n}=3$ for each group). dpf, days post-fertilization; Ter, terfenadine; FMS, fimasartan. ${ }^{a} \mathrm{p}<0.05,{ }^{\mathrm{b}} \mathrm{p}<0.01$.

decrease in blood flow velocity induced by terfenadine.

\section{Fimasartan improves atrioventricular dyssynchrony}

We previously reported AV dyssynchrony in zebrafish after treatment with terfenadine [15]. We investigated contraction timing, rhythmic rate, and atrial area to confirm whether fimasartan improved terfenadine-induced AV dyssynchrony. The atria and ventricles of $\mathrm{Tg}$ (cmlc2:gfp) larvae were clearly visible, and contraction times were discriminated by using a series of images taken by in vivo time-lapse video recordings. In contrast with the synchronous contraction and dilatation of the atria and ventricles observed in vehicle-treated larvae, the larvae treated with terfenadine showed dyssynchronous AV movement (Fig. $3 \mathrm{~A}$ and Supplementary Video 1). The AV ratio of terfenadine-treated larvae was approximately 0.6 , and the atrial area was significantly increased. Unexpectedly, in larvae treated with fimasar- tan, atrial contraction was more regular and the AV ratio was preserved, with a value of approximately o.8 (Fig. 3B). The atrial area was also significantly smaller than that in the terfenadine-treated group. The atrial areas of terfenadine-treated larvae were increased four-fold compared with the vehicle-treated larvae, from 9,080.2 \pm $1,646.6$ to $36,293.8 \pm 2,322.5 \mu \mathrm{m}^{2}$, whereas the larvae treated with fimasartan only showed a two-fold increase, to $18,500.8 \pm 807.4 \mu \mathrm{m}^{2}$ (Fig. $3 \mathrm{C}$ ).

\section{Fimasartan prevents apoptotic cell death}

To evaluate whether fimasartan reduced apoptotic cell death in terfenadine-induced zebrafish $\mathrm{HF}$, an in situ TUNEL assay was performed to visualize apoptotic cells. Increased chromatin fragmentation, indicating cell death, was observed around the heart field in terfenadine-treated larvae, and this was suppressed by fimasartan treatment (Fig. 4A-4C). The quantification of 


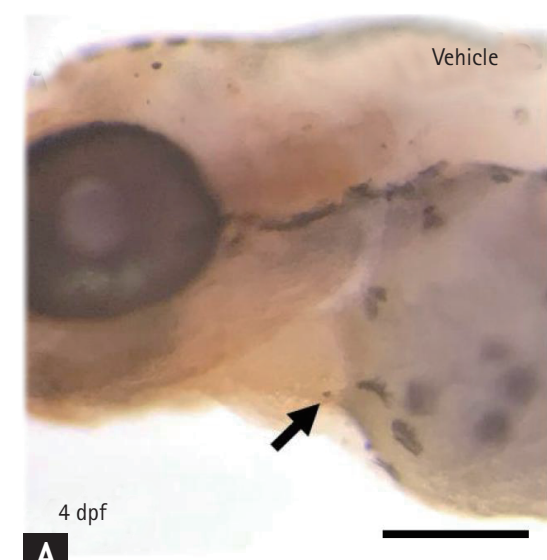

A

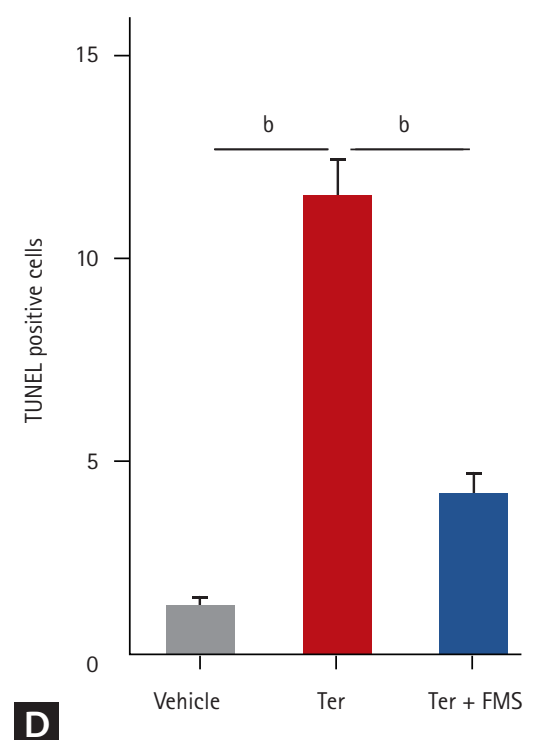

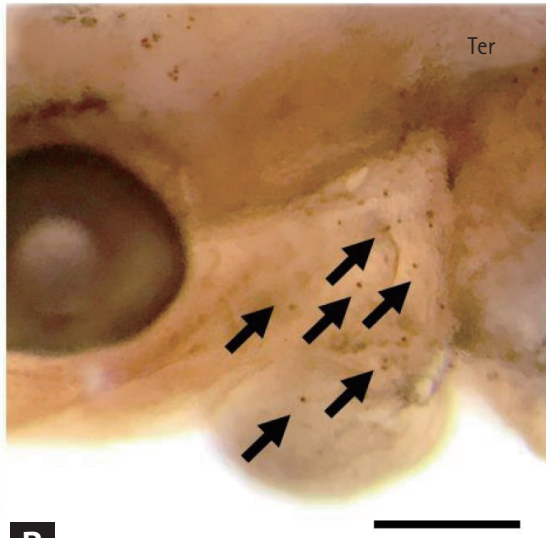

B

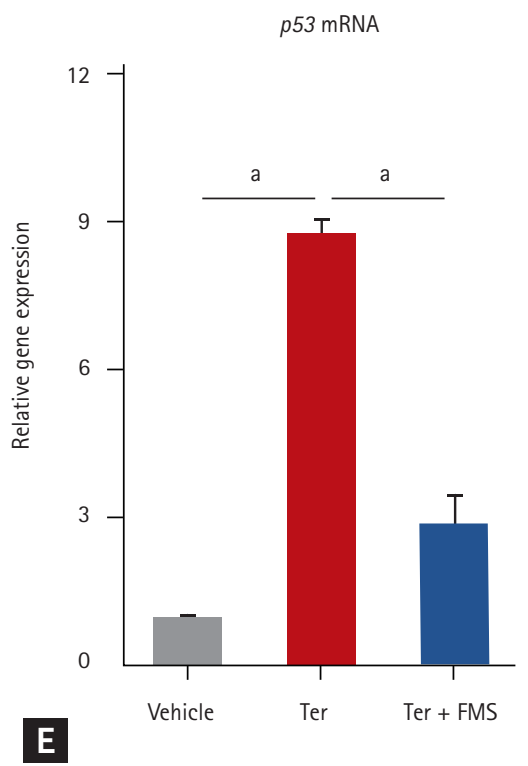

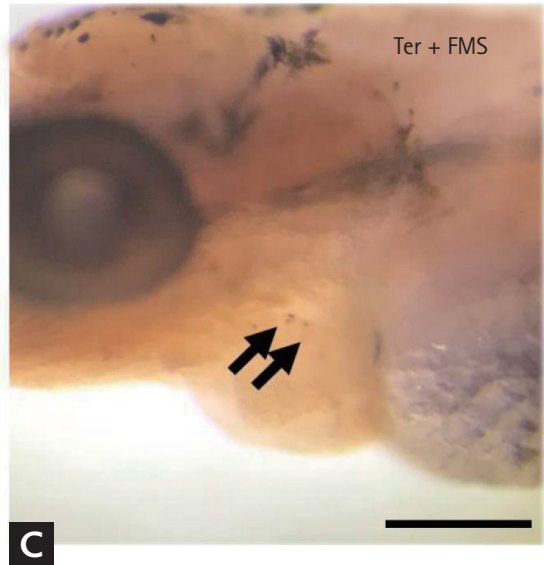

Figure 4. Fimasartan prevents apoptotic cell death. (A-C) Whole-mount TUNEL staining of zebrafish larvae. (A) Brown-color (arrows) indicates TUNEL-positive apoptotic cells. (B) Apoptotic cells are dramatically increased in terfenadine-treated larvae, (C) while treatment with fimasartan effectively decreased apoptotic cells. (D) Quantification of TUNEL-positive cells ( $\mathrm{n}=4$ for each group). (E) Levels of p53 mRNA expression ( $n=20$ for each group). Scale bar $=1 \mathrm{~mm}$. Error bars represent standard deviation. dpf, days post-fertilization; Ter, terfenadine; FMS, fimasartan. ${ }^{\mathrm{a}} \mathrm{p}<$ $0.01,{ }^{b} p<0.001$.
TUNEL-positive cells also yielded similar results, which showed that fimasartan suppressed chromatin fragmentation induced by terfenadine (Fig. 4D). Transcription of the 553 gene was also significantly increased in terfenadine-treated larvae, whereas fimasartan-treated larvae showed only a mild increase in p53 mRNA expression. These findings indicated that fimasartan significantly suppressed terfenadine-induced apoptosis in an in vivo zebrafish model of HF.

\section{Fimasartan improves zebrafish motility and survival}

The motility of zebrafish larvae was dramatically reduced by terfenadine treatment, but showed near-normal values in larvae treated with fimasartan. The quantification of motility by using video analysis showed a significant difference between terfenadine-treated and fimasartan-treated larvae $(9.58 \pm 6.89 \mathrm{~mm}$ vs. $36.97 \pm 22.79 \mathrm{~mm}, \mathrm{p}$
$<0.001$ ) (Fig. 5A-C and Supplemental Video 2). Fimasartan treatment also significantly reduced the mortality of terfenadine-treated zebrafish (terfenadine + fimasartan $36.0 \%$ vs. terfenadine $96.0 \%, p<0.001$ ) (Fig. ${ }_{5} \mathrm{D}$ ).

\section{DISCUSSION}

In this study, treatment with fimasartan effectively prevented the development of $\mathrm{HF}$ and improved survival in our zebrafish model. First, HF was induced by treatment of terfenadine for 12 hours, as denoted by marked dilatation of cardiac chambers and decreased ventricular systolic function; in contrast, treatment with fimasartan reversed changes in heart size and enhanced its function. Second, fimasartan treatment effectively preserved cardiac output, reduced heartbeat variability, accelerated 


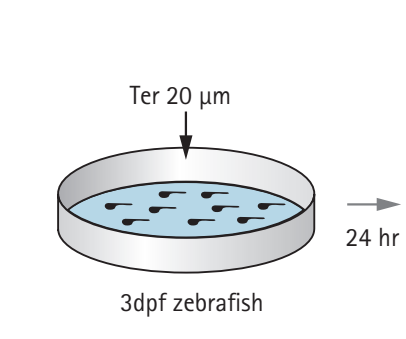

A

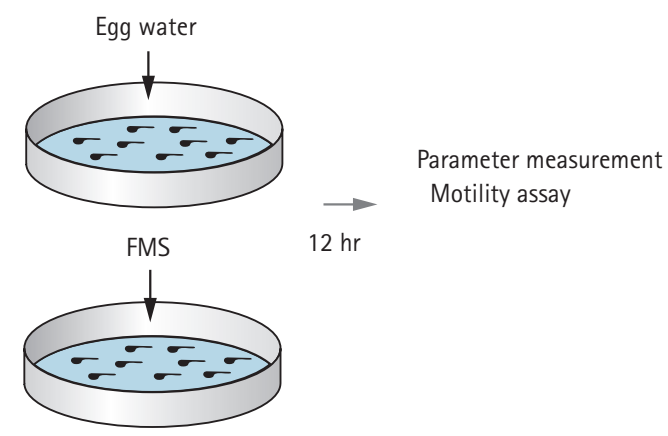

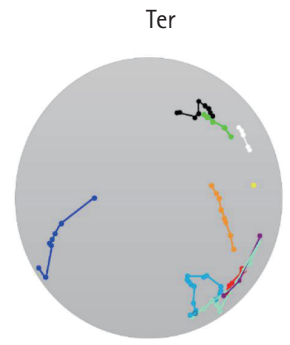

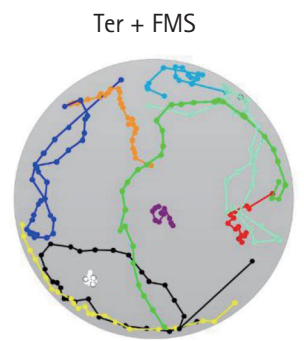

C

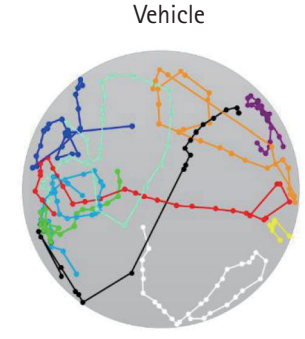

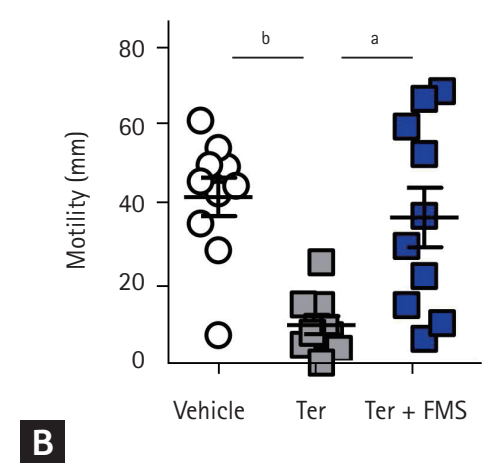

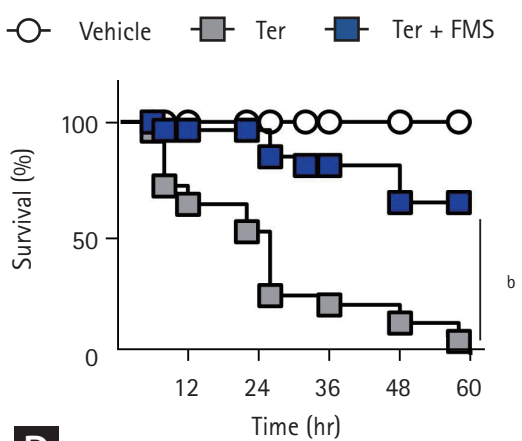

D

Figure 5. Fimasartan improves motility and survival. (A) Mobility study design. (B) Distance travelled by zebrafish larvae (n = 10 for each group). (C) Movement trajectories of zebrafish larvae $(n=10)$. Each color represents the trajectories of zebrafish larvae over 20 seconds. (D) Survival curve of zebrafish larvae ( $n=25$ for each group). Ter, terfenadine; dpf, days post-fertilization; FMS, fimasartan. ${ }^{a} p<0.01,{ }^{b} p<0.001$.

blood flow, and decreased atrial area. Finally, fimasartan treatment also improved mobility and the survival of terfenadine-treated zebrafish. These results suggested that fimasartan may be a candidate pharmacotherapy agent for HF.

In the current study, an in vivo zebrafish model of terfenadine-induced cardiomyopathy was used to examine the protective effects of fimasartan in HF. By using this model, we were able to assess the entire scope of HF pathophysiology. At the cellular level, the mRNA expression of natriuretic peptide was congruent with treatment status: it was increased in terfenadine-treated zebrafish, but was decreased by fimasartan treatment. The physiologic status was assessed by the time interval between heartbeats and the mean blood flow velocity. We were also able to assess functional status through the measurement of FS and motility by using video analysis. Finally, p53 mRNA expression and chromatin fragmentation by TUNEL assay was used to assess cell survival.
Whenever cardiac output falls, compensatory mechanisms are induced in an attempt to maintain blood pressure and organ perfusion. For this reason, RAAS is a well-established therapeutic target in the treatment of HF. However, angiotensin II receptor antagonists are recommended only when ACE inhibitors are intolerable or contraindicated. Previous clinical studies have shown that losartan [18], valsartan [19], and candesartan [20] may improve outcomes and reduce hospitalization in patients HF; however, there have been no studies on the use of fimasartan.

Previous studies have reported that terfenadine induces cardiac toxicity with bradycardia and arrhythmia in zebrafish larvae $[12,21,22]$. Moreover, recent studies have confirmed that terfenadine-treated zebrafish larvae can be used as a model to screen for positive inotropes [13]. The features of cardiac dysfunction in zebrafish larvae induced by terfenadine treatment are similar to those in human HF. In addition to triggering atrioventricu- 
lar dyssynchrony and venous congestion in zebrafish, terfenadine has also been reported to cause ventricular tachycardia and fibrillation in rabbits [23], which may result in the rapid decline of ventricular function and the development of $\mathrm{HF}$ [24]. In the current study, we treated zebrafish larvae at $3 \mathrm{dpf}$ with $20 \mu \mathrm{M}$ terfenadine for 12 hours to induce dilated cardiomyopathy. As expected, the larvae showed an increase in cardiac dimensions and a decrease in systolic function. In addition, blood flow velocity was decreased and contraction time was delayed. This decrease in blood flow can result in hypoperfusion, and there have been several reports that renal hypoperfusion activates the RAA system in patients with HF [25-27]. Reduced cardiac output in early HF induces RAAS-activated fluid retention, which subsequently increases ventricular preload and cardiac output as a compensation method. However, if ventricular dysfunction progresses, compensation mechanisms fail and venous pressure increases [28]. As we have demonstrated similar mechanisms in zebrafish larvae, we believe that our terfenadine-induced model of dilated cardiomyopathy can be used to test the effectiveness of RAAS inhibitors.

Fimasartan, an ARB, has been approved for the treatment of essential hypertension in Korea. In addition to its angiotensin II-blocking effects, fimasartan has shown superior inhibition of the contraction of isolated rabbit thoracic aorta compared with other ARBs, such as losartan and candesartan [29], and has been reported to have protective effects in a porcine model of acute myocardial infarction [30]. Fimasartan was also reported to reduce ischemic cell death when used to treat transient focal ischemia in rats [31], and to prevent unilateral ureteral obstruction-induced apoptosis in mice [32]. Although fimasartan has not been previously studied for the treatment of $\mathrm{HF}$, it is expected to exert similar effects to other ARBs, and potentially greater efficacy because of its higher affinity for angiotensin II type 1 (AT1) receptors compared with other drugs of its class in preclinical studies [33]. The AT1 receptor is involved in the classical physiological actions of angiotensin II: the regulation of blood pressure, electrolytes, and water balance; thirst; hormone secretion, and the regulation of renal function [34]. Therefore, the inhibition of angiotensin II signals can dilate arteries and veins, thereby reducing arterial pressure, decreasing preload and afterload, and increasing blood flow velocity and FS.
In line with previous studies that showed the potent attenuation of myocardial apoptotic cell death by fimasartan in reperfused rat hearts and $\mathrm{H}_{9} \mathrm{C} 2$ cells [10], we showed that treatment with fimasartan prevented apoptotic cell death. The activation of the AT1 receptor enhances the influx of intracellular calcium and stimulates calcium-dependent endogenic endonucleases, which cause DNA laddering, cell shrinkage, and the formation of apoptotic bodies [10]. In addition, AT1 receptor blockers may exert cardioprotective actions in addition to their ability to lower blood pressure, such as anti-apoptotic, anti-atherosclerotic, and target organ-protecting effects [35]. Our study also showed that blocking the AT1 receptor decreased the number of apoptotic cells and pro-apoptotic p53 expression in zebrafish larvae (Fig. 4). Interestingly, $\mathrm{p}_{53}$ induces apoptosis in cardiac myocytes via the activation of classical renin-angiotensin system. This may be limited to myocytes because the induction of $\mathrm{p}_{53}$ is insufficient to trigger apoptosis in other cell types [36].

Randomized trials have shown that ARBs improve survival in patients with HF who are intolerable to ACE inhibitors [37-39]. However, there have been reports that elderly patients with HF treated with losartan had poorer survival rates than those treated with other commonly used ARBs [40]. The current guidelines state that only losartan, valsartan, and candesartan have effects comparable with ACE inhibitors in patients with advanced HF, but should only be used as a second-line treatment for patients intolerant to ACE inhibitors. In our study, treatment with fimasartan significantly increased zebrafish survival and functional status (Fig. 5). The in vivo results of this animal study suggested that human clinical studies are warranted to further evaluate the efficacy of fimasartan in patients with $\mathrm{HF}$.

The limitation of this current study is that although zebrafish hearts have similar features to human hearts, they only have a single atrium and ventricle. Furthermore, our study evaluated terfenadine treatment-related HF for 4 days, and that a longer period of observation may be necessary to evaluate the full scope of HF.

Fimasartan prevented HF symptoms and modified physiologic changes in an in vivo zebrafish model of terfenadine-induced HF. Furthermore, fimasartan also decreased the number of apoptotic cells and increased zebrafish survival and motility. Human studies are war- 
ranted for further evaluation of the role of fimasartan in the treatment of HF.

\section{KEY MESSAGE}

1. Zebrafish exposed to terfenadine showed changes similar to that of human heart failure (HF).

2. Treatment with fimasartan, an angiotensin receptor blocker, prevented symptoms and modified physiologic changes in an in vivo zebrafish model of HF.

3. Fimasartan could be a candidate drug for human HF, and further evaluations are warranted.

\section{Conflict of interest}

No potential conflict of interest relevant to this article was reported.

\section{Acknowledgments}

This study was supported by a grant from the National Research Foundation of Korea (2017015015) and the Education and Research Encouragement Fund of Seoul National University Hospital (03-2016-0290).

\section{REFERENCES}

1. Ambrosy AP, Fonarow GC, Butler J, et al. The global health and economic burden of hospitalizations for heart failure: lessons learned from hospitalized heart failure registries. J Am Coll Cardiol 2014;63:1123-1133.

2. Writing Group Members, Mozaffarian D, Benjamin EJ, et al. Executive summary: heart disease and stroke statistics. 2016 Update: a report from the American Heart Association. Circulation 2016;133:447-454.

3. Lee JH, Lim NK, Cho MC, Park HY. Epidemiology of heart failure in Korea: present and future. Korean Circ J 2016;46:658-664.

4. Lee SE, Cho HJ, Lee HY, et al. A multicentre cohort study of acute heart failure syndromes in Korea: rationale, design, and interim observations of the Korean Acute Heart Failure (KorAHF) registry. Eur J Heart Fail 2014;16:700708.

5. Lee SE, Lee HY, Cho HJ, et al. Clinical characteristics and outcome of acute heart failure in Korea: results from the Korean Acute Heart Failure Registry (KorAHF). Korean Circ J 2017;47:341-353.

6. Caldeira D, David C, Sampaio C. Tolerability of angiotensin-receptor blockers in patients with intolerance to angiotensin-converting enzyme inhibitors: a systematic review and meta-analysis. Am J Cardiovasc Drugs 2012;12:263-277.

7. Lee HY, Oh BH. Fimasartan: a new angiotensin receptor blocker. Drugs 2016;76:1015-1022.

8. Lee H, Kim KS, Chae SC, Jeong MH, Kim DS, Oh BH. Ambulatory blood pressure response to once-daily fimasartan: an 8-week, multicenter, randomized, double-blind, active-comparator, parallel-group study in Korean patients with mild to moderate essential hypertension. Clin Ther 2013;35:1337-1349.

9. Lee SE, Kim YJ, Lee HY, et al. Efficacy and tolerability of fimasartan, a new angiotensin receptor blocker, compared with losartan (50/100 mg): a 12-week, phase III, multicenter, prospective, randomized, double-blind, parallel-group, dose escalation clinical trial with an optional 12-week extension phase in adult Korean patients with mild-to-moderate hypertension. Clin Ther 2012;34:552568.

10. Han J, Park SJ, Thu VT, et al. Effects of the novel angiotensin II receptor type I antagonist, fimasartan on myocardial ischemia/reperfusion injury. Int J Cardiol 2013;168:2851-2859.

11. Lee JY, Lee CW, Kim WJ, et al. Antiatherosclerotic effects of the novel angiotensin receptor antagonist Fimasartan on plaque progression and stability in a rabbit model: a double-blind placebo-controlled trial. J Cardiovasc Pharmacol 2013;62:229-236.

12. Chang SA, Lim BK, Lee YJ, Hong MK, Choi JO, Jeon ES. A novel angiotensin type I receptor antagonist, fimasartan, prevents doxorubicin-induced cardiotoxicity in rats. J Korean Med Sci 2015;30:559-568.

13. Tang C, Xie D, Feng B. Zebrafish as a new model for phenotype-based screening of positive inotropic agents. Chem Biol Drug Des 2015;85:253-258.

14. Huang CC, Monte A, Cook JM, Kabir MS, Peterson KP. Zebrafish heart failure models for the evaluation of chemical probes and drugs. Assay Drug Dev Technol 2013;11:561-572.

15. Gu G, Na Y, Chung H, Seok SH, Lee HY. Zebrafish larvae model of dilated cardiomyopathy induced by terfenadine. 
Korean Circ J 2017;47:960-969.

16. Westerfield M. The Zebrafish Book: A Guide for the Laboratory Use of Zebrafish (Danio rerio). Eugene (OR): University of Oregon, 2000.

17. Huang CJ, Tu CT, Hsiao CD, Hsieh FJ, Tsai HJ. Germ-line transmission of a myocardium-specific GFP transgene reveals critical regulatory elements in the cardiac myosin light chain 2 promoter of zebrafish. Dev Dyn 2003;228:3040.

18. Pitt B, Poole-Wilson PA, Segal R, et al. Effect of losartan compared with captopril on mortality in patients with symptomatic heart failure: randomised trial. The Losartan Heart Failure Survival Study ELITE II. Lancet 2000;355:1582-1587.

19. Cohn JN, Tognoni G; Valsartan Heart Failure Trial Investigators. A randomized trial of the angiotensin-receptor blocker valsartan in chronic heart failure. N Engl J Med 2001;345:1667-1675.

20. McMurray JJ, Ostergren J, Swedberg K, et al. Effects of candesartan in patients with chronic heart failure and reduced left-ventricular systolic function taking angiotensin-converting-enzyme inhibitors: the CHARM-Added trial. Lancet 2003;362:767-771.

21. Hassel D, Scholz EP, Trano N, et al. Deficient zebrafish ether-à-go-go-related gene channel gating causes shortQT syndrome in zebrafish reggae mutants. Circulation 2008;117:866-875.

22. Milan DJ, Peterson TA, Ruskin JN, Peterson RT, MacRae CA. Drugs that induce repolarization abnormalities cause bradycardia in zebrafish. Circulation 2003;107:1355-1358.

23. Lu HR, Hermans AN, Gallacher DJ. Does terfenadine-induced ventricular tachycardia/fibrillation directly relate to its QT prolongation and Torsades de Pointes? Br J Pharmacol 2012;166:1490-1502.

24. Gopinathannair R, Etheridge SP, Marchlinski FE, Spinale FG, Lakkireddy D, Olshansky B. Arrhythmia-induced cardiomyopathies: mechanisms, recognition, and management. J Am Coll Cardiol 2015;66:1714-1728.

25. Andrew P. Renin-angiotensin-aldosterone activation in heart failure, aldosterone escape. Chest 2002;122:755.

26. Viswanathan G, Gilbert S. The cardiorenal syndrome: making the connection. Int J Nephrol 2010;2011:283137.

27. Bernstein KE, Giani JF, Shen XZ, Gonzalez-Villalobos RA. Renal angiotensin-converting enzyme and blood pressure control. Curr Opin Nephrol Hypertens 2014;23:106112.
28. Guyton AC, Hall JE. Textbook of Medical Physiology. Philadelphia (PA): WB Saunders, 2000:392-401.

29. Kim JH, Lee JH, Paik SH, Kim JH, Chi YH. Fimasartan, a novel angiotensin II receptor antagonist. Arch Pharm Res 2012;35:1123-1126.

30. Sim DS, Jeong MH, Song HC, et al. Cardioprotective effect of fimasartan, a new angiotensin receptor blocker, in a porcine model of acute myocardial infarction. J Korean Med Sci 2015;30:34-43.

31. Kim CK, Yang XL, Kim YJ, et al. Effect of long-term treatment with fimasartan on transient focal ischemia in rat brain. Biomed Res Int 2015;2015:295925.

32. Kim S, Kim SJ, Yoon HE, et al. Fimasartan, a novel angiotensin-receptor blocker, protects against renal inflammation and fibrosis in mice with unilateral ureteral obstruction: the possible role of Nrf2. Int J Med Sci 2015;12:891904 .

33. Paik SH, Chi YH, Lee JH, Han HS, Lee KT. Pharmacological profiles of a highly potent and long-acting angiotensin II receptor antagonist, fimasartan, in rats and dogs after oral administration. Biol Pharm Bull 2017;40:992-1001.

34. Kaschina E, Unger T. Angiotensin AT1/AT2 receptors: regulation, signaling and function. Blood Press 2003;12:70-88.

35. Munger MA. Use of Angiotensin receptor blockers in cardiovascular protection: current evidence and future directions. P T 2011;36:22-40.

36. Pierzchalski P, Reiss K, Cheng W, et al. p53 Induces myocyte apoptosis via the activation of the renin-angiotensin system. Exp Cell Res 1997;234:57-65.

37. Maggioni AP, Anand I, Gottlieb SO, et al. Effects of valsartan on morbidity and mortality in patients with heart failure not receiving angiotensin-converting enzyme inhibitors. J Am Coll Cardiol 2002;40:1414-1421.

38. Pfeffer MA, Swedberg K, Granger CB, et al. Effects of candesartan on mortality and morbidity in patients with chronic heart failure: the CHARM-Overall programme. Lancet 2003;362:759-766.

39. Lee VC, Rhew DC, Dylan M, Badamgarav E, Braunstein GD, Weingarten SR. Meta-analysis: angiotensin-receptor blockers in chronic heart failure and high-risk acute myocardial infarction. Ann Intern Med 2004;141:693-704.

40. Hudson M, Humphries K, Tu JV, Behlouli H, Sheppard $\mathrm{R}$, Pilote L. Angiotensin II receptor blockers for the treatment of heart failure: a class effect? Pharmacotherapy 2007;27:526-534. 


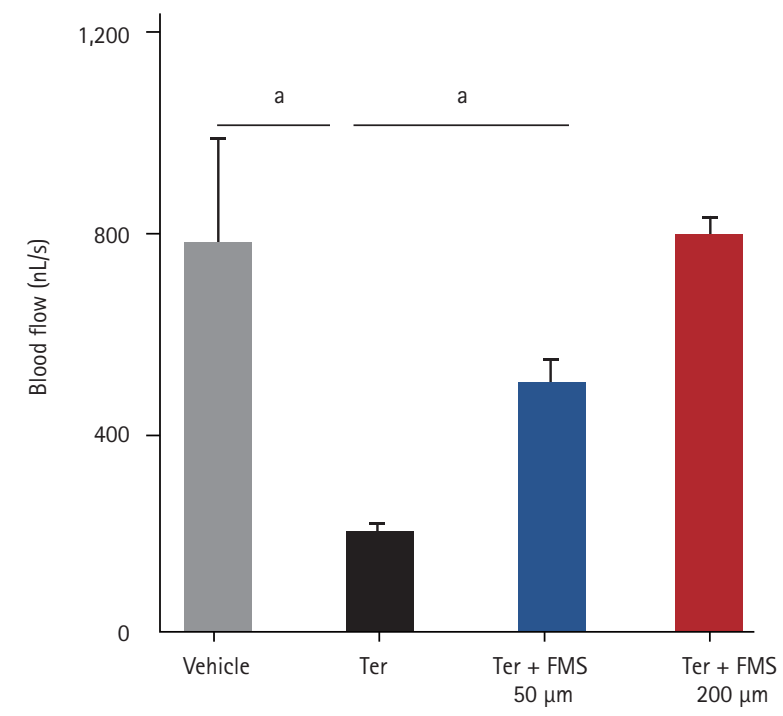

Supplementary Figure 1. Blood flow velocity according to fimasartan dose. Ter, terfenadine; FMS, fimasartan. ${ }^{a} p<0.05$. 


\section{SUPPLEMENTARY MATERIAL}

Supplementary Video 1. Fimasartan treatment improves cardiac contractility and atrioventricular (AV) synchrony of zebrafish. Time lapse video recording using Tg (cmlc2: gfp) zebrafish treated with (A) vehicle, (B) terfenadine (Ter), and (C) Ter + fimasartan (FMS).
Supplementary Video 2. Fimasartan (FMS) treatment improved motility of zebrafish. Motility of zebrafish treated with (A) vehicle, (B) terfenadine (Ter), and (C) Ter + fimasar$\tan (\mathrm{FMS})$ (recorded for 20 seconds). 